\title{
Fasciolíase: relato de dois casos em área rural do Rio de Janeiro
}

\author{
Fascioliasis: report of two cases from rural areas of Rio de Janeiro
}

\author{
Ricardo Pereira Igreja ${ }^{1}$, Magali Gonçalves Muniz Barreto ${ }^{2}$ \\ e Marisa da Silveira Soares ${ }^{2}$
}

\begin{abstract}
RESUMO
São descritos dois casos de fasciolíase em áreas rurais do Rio de Janeiro, endêmicas para esquistossomose, sendo ambos surpreendidos durante inquéritos coprológicos. 0 paciente de Paracambi queixava-se de tonteira. A paciente de Sumidouro queixava-se de tonteira, cansaço e tosse, tendo sido tratada com praziquantel; seus exames de controle foram negativos.
\end{abstract}

Palavras-chaves: Fasciolíase. Zoonose. Paracambi. Sumidouro.

\begin{abstract}
We describe two cases of fascioliasis from rural areas of Rio de Janeiro that are endemic for schistosomiasis, both of which were found during a coprological survey. The patient from Paracambi complained of dizziness. The patient from Sumidouro complained of dizziness, tiredness and cough. She was treated with praziquantel and her stool parasitological examination became negative.
\end{abstract}

key-words: Fasciolíasis. Zoonosis. Paracambi. Sumidouro.

Afasciolíase éuma zoonose causada por um trematódeo parasito de herbívoros, a Fasciola hepatica, e que acomete principalmente 0 fígado, a vesícula biliar e as vias biliares². Os ovos, que são levados pela bile ao intestino e eliminados pelas fezes, eclodem ao ganhar 0 ambiente aquático, dando origem aos miracídios. Estes infectam 0 hospedeiro intermediário, um molusco do gênero Lymnaea, que liberará posteriormente as cercárias, que evoluem para cistos. Tais cistos, que passam a ser denominados metacercárias, localizam-se agora na superfície de plantas aquáticas, como 0 agrião. Quando ingeridos pelo hospedeiro definitivo, as metacercárias são liberadas na porção superior do intestino, alcançam a cavidade peritoneal e penetram no fígado através da cápsula de Glisson, aonde atingirão os ductos biliares e se tornarão adultos².
Até recentemente, a fasciolíase humana era considerada uma zoonose secundária. Entretanto, a importância da doença humana ao nível de saúde pública aumentou nos últimos anos. Em 25 anos foram descritos 7.071 casos da doença, em 51 países de todos os continentes, sendo o maior número de casos, 3627, no continente american $0^{8}$. Estimase que possam existir até 17 milhões de pessoas infectadas com Fasciola hepatica no mund0 ${ }^{8}$.

No Brasil, foram descritos 57 casos da doença em seres humanos, nos Estados do Paraná (a grande maioria), São Paulo, Rio de Janeiro, Minas Gerais, Mato Grosso do Sul, Bahia, Rio Grande do Sul e Santa Catarina ${ }^{2}$. Há um único relato, de dois casos, no Estado do Rio de Janeiro ${ }^{10}$.

1. Faculdade de Medicina da Universidade Federal do Rio de Janeiro e da Escola de Medicina e Cirurgia da Universidade Federal do Estado do Rio de Janeiro, Rio de Janeiro, RJ, Brasil. 2. Laboratório de Avaliação e Promoção da Saúde Ambiental do Departamento de Biologia do Instituto Oswaldo Cruz da Fundação Oswaldo Cruz, Rio de Janeiro, RJ.

Aprovado pelo Comitê de Ética em Pesquisa da Fundação Oswaldo Cruz. Apoio: PAPES / FIOCRUZ e CNPq

Endereço para correspondência: Dra. Magali Gonçalves Muniz Barreto. Dept ${ }^{\circ}$ de Biologia/FIOCRUZ. Av. Brasil 4365, Pavilhão Lauro Travassos, Manguinhos. 21045-900 Rio de Janeiro, RJ.

e-mail: mbarreto@ cives.ufrj.br

Tel: 21 2560-6474

Recebido para publicação em 24/5/2004

Aceito em 10/7/2004 


\section{RELATO DOS CASOS}

São descritos dois casos de fasciolíase ocorridos em área rural do Estado do Rio de Janeiro onde há limneídeos. Nos últimos 12 anos, nossa equipe tem realizado inquéritos coprológicos nos municípios de Sumidouro e Paracambi, em busca de casos de esquistossomose. Foram realizados cerca de 20.000 exames em 4.000 pessoas (algumas delas examinadas mais de uma vez) pelos métodos de Hoffman cols ${ }^{5}$ e Kato-Katz ${ }^{6}$, com até três amostras por pessoa obtidas em dias diferentes e 4 lâminas por amostra. 0 primeiro caso, diagnosticado em 2001, em Paracambi, era um agricultor de 32 anos, com história de etilismo, positivo em duas amostras, que se queixava de tonteiras e recusou o tratamento para a fasciolíase. Apresentava, também, ancilostomíase. 0 outro caso foi diagnosticado em 2003, em Sumidouro, em uma das amostras de fezes de uma dona de casa de 48 anos, com hábito de comer vegetais crus cultivados no município e que criava bovinos e outros animais. Queixava-se de tonteira, cansaço e tosse, mas no exame físico apresentava apenas obesidade e varizes. 0 caso foi tratado com praziquantel, na dose de $75 \mathrm{mg} / \mathrm{kg}$ dividida em três tomadas (em um único dia). Os 3 exames parasitológicos de controle foram negativos. Não pode ser descartada a hipótese de ovos do parasita estarem apenas passando pelo trato intestinal.

\section{DISCUSSÃ0}

Relatamos dois indivíduos com queixas inespecíficas, compatíveis com fasciolíase, mas sem os sintomas mais clássicos da doença, ao contrário da maior parte dos relatos de caso, que descrevem pacientes com as formas graves de fasciolíase ${ }^{7}$. Resultados falsos positivos poderiam ocorrer quando 0 paciente, antes da realização do exame, ingerisse fígado ou outros alimentos que pudessem conter extratos hepáticos e biliares contaminados ${ }^{11}$. Nós não dispomos de tal informação dos nossos pacientes. Todavia, ambos moravam em áreas rurais, em contato com bovinos, e a paciente tinha 0 hábito de comer vegetais crus, condições favoráveis à transmissão da doença ${ }^{1}{ }^{8}$.

Aapresentação clínica da infecção pela Fasciola hepatica se dá sob uma forma aguda e outra crônica. A fase aguda inicia poucos dias após a ingestão de metacercárias e os sintomas apresentados são febre, dor abdominal, cefaléia, prurido e urticária. Após 2 a 4 meses a fase biliar crônica substitui gradualmente a fase aguda. 0 paciente pode tornarse assintomático ou ter episódios de cólica biliar, dor abdominal e fadiga, que podem persistir por muitos anos ${ }^{7}$. A confirmação diagnóstica é realizada através da visualização dos ovos do parasita nas fezes ou no suco duodenal e também através de métodos imunológicos ${ }^{3}$.

De acordo com Haswell-Elkins e Elkins, como a fasciolíase tem sido descrita principalmente em relatos de caso, mais do que em estudos baseados em comunidades, é difícil avaliar a extensão da infecção nas áreas em que os casos foram relatados ${ }^{4}$. Tal raciocínio não se aplica aos nossos pacientes, já que os diagnósticos foram realizados através de amplos estudos coprológicos das populações. As razões que explicariam a baixa freqüência da infecção no Brasil seriam a subnotificação, a falta de diagnóstico ou mesmo o fato da doença ser realmente rara ${ }^{1}$.

Ainda não existe tratamento específico que tenha apresentado resultados satisfatórios ${ }^{2}$, sendo utilizado 0 bithionol, a deidroemetina e 0 triclabendazo ${ }^{3}{ }^{7}$. Embora 0 praziquantel tenha uma eficácia controversa ${ }^{3}$, optamos pelo seu uso devido à falta de acesso imediato às demais medicações e obtivemos sucesso.

Este relato descreve duas novas áreas de fasciolíase no Estado do Rio de Janeiro, endêmicas para esquistossomose, a partir de pacientes surpreendidos durante inquéritos coprológicos, sem sinais de gravidade da doença.

\section{AGRADECIMENTOS}

Marli Tex Paulino, Susana Balmant Emerique Simões e Weslley Rodrigues Abreu pela leitura das lâminas.

\section{REFERÊNCIAS BIBLIOGRÁFICAS}

1. Andrade Neto JL, Carneiro Filho M, Luz E, Siciliano RF, Oliveira Filho AG, Pisani JC. Human Fascioliasis in The Metropolitan Area of Curitiba, Brazil - Evaluation of The Foci of Infection and Report of Nine Cases Treated with Triclabendazole. The Brazilian Journal of Infectious Diseases 3:220-225, 1999.

2. Farinazzo RJM, Igreja RP, Huggins DW. Fasciolíase Hepática. In: Batista RS, Gomes AP, Igreja RP, Huggins DW ( eds) Medicina Tropical. Abordagem atual das Doenças Infecciosas e Parasitárias. Editora Cultura Médica, Rio de Janeiro, p.287-290, 2001.

3. Gentilini M. Médecine Tropicale, 5e édition, Editora Flamarion, Paris, 1995.

4. Haswell-Elkins MR, Elkins D. Food-Borne Trematodes. In: Cook GC ( ed) Manson's Tropical Diseases, 20 $0^{\text {th }}$ edition, WB Saunders Co, London, $\mathrm{p}$. 1457-1476, 1996.

5. Hoffmann WA, Pons J A, Janer JL. The sedimentation concentration method in schistosomiasis mansoni. Puerto Rico Journal of Public Health and Tropical Medicine 9:283-291, 1934

6. Katz N, Chaves A, Pellegrino J. A simple device for quantitative stool thicksmear technique in schistosomiasis mansoni. Revista do Instituto de Medicina Tropical de São Paulo 14:397-400, 1972

7. Lee Haris N, McNeely WF, Shepard JO, Ebeling SH, Ellender SM, Peters CC. Weekly Clinicopathological Exercises. The New England Journal of Medicine 346:1232-1239, 2002.

8. Mas-Coma MS, Esteban JG, Bargues MD. Epidemiology of human fascioliasis: a review and proposed new classification. Bulletin of the World Health Organization 77:340-346, 1999.

9. Mezzari A, Antunes HBB, Coelho N, Cauduro PF, Brodt TC. Fasciolíase humana no Brasil diagnosticada por colangiografia endoscópica retrógrada. Jornal Brasileiro de Patologia 36:93-95, 2000.

10. Pile E, Gazeta G, Santos JAA, Coelho B, Serra-Freire NM. Ocorrência de fascioliasis humana no município de Volta Redonda, RJ, Brasil. Revista de Saúde Pública 34:413-414, 2000.

11. Rey L. Bases da Parasitologia Médica. Guanabara Koogan, Rio de Janeiro, 2002. 